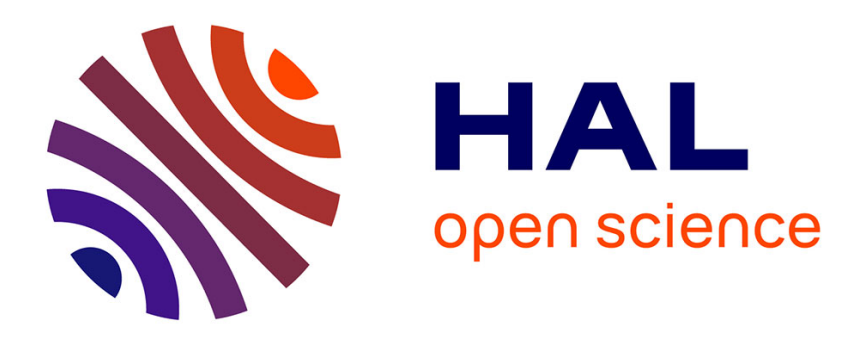

\title{
The Percolation Model for Rapid Crack Propagation
}

\author{
M. Watanabe, S. Shudoh, S. Katsura
}

\section{To cite this version:}

M. Watanabe, S. Shudoh, S. Katsura. The Percolation Model for Rapid Crack Propagation. Journal de Physique IV Proceedings, 1996, 06 (C6), pp.C6-403-C6-409. 10.1051/jp4:1996640 . jpa-00254473

\section{HAL Id: jpa-00254473 https://hal.science/jpa-00254473}

Submitted on 1 Jan 1996

HAL is a multi-disciplinary open access archive for the deposit and dissemination of scientific research documents, whether they are published or not. The documents may come from teaching and research institutions in France or abroad, or from public or private research centers.
L'archive ouverte pluridisciplinaire HAL, est destinée au dépôt et à la diffusion de documents scientifiques de niveau recherche, publiés ou non, émanant des établissements d'enseignement et de recherche français ou étrangers, des laboratoires publics ou privés. 


\title{
The Percolation Model for Rapid Crack Propagation
}

\author{
M. Watanabe, S. Shudoh* and S. Katsura** \\ KinKi University, Faculty of Engineering, Takaya, Higashi-Hiroshima 739-21, Japan \\ * Mitsubishi Denki Control Software, 6-1-2 Hamayama-douri, Hyogo-Ku, Kobe 652, Japan \\ ** Nippon Denki Robot Engineering, 1-1-25 Shinurashima-cho Kanagawa-ku, Yokohama 221, Japan
}

\begin{abstract}
The percolation model for rapid crack propagation is proposed and it's properties are examined. In this model, a crack progresses through a two dimensional square lattice when initially closed "bond", defined at each square, is broken. The probability $p$ of breaking a bond is defined as $p=K / \sqrt{R}$ for $1 \leq R \leq R$, where $\mathrm{K}(0<\mathrm{K} \leq 1)$ is a constant and $\mathrm{R}$ is a row or column distance from the "broken bond", which remains active during an unit time. $R_{I C}$ is the critical distance within which the bond breaks with the probability p. The fractal properties of fractured patterns starting from a single broken bond according to this rule is studied. The initially plane crack tip progresses through $60 \times 270$ square lattice, and the velocity of a crack for various values of $\mathrm{K}$ and $\mathrm{R}_{\mathrm{IC}}$ are investigated. Implication of this simulation to the experiment of rapid crack propagation (RCP) is discussed.
\end{abstract}

\section{INTRODUCTION}

Although much work has been done on rapid crack propagation (RCP) for many years, the mechanisms that govern the dynamics of a crack are not well understood. The recent experiment of RCP in PMMA, performed by Fineberg et. al. [1], shows that the velocity of a crack oscillates when the velocity exceeds the critical velocity. The cause of the oscillation is found as an attempted local crack branching [2]. In a single edge notch tensile (SENT) experiment of RCP in PMMA and polycarbonate(PC), Ivankovic and Williams [3] have observed that the velocity of a crack is constant, while the fracture surface remains smooth. They suggest that the low local Young's modulus at the tip of a crack plays an important role in RCP from the combined analysis of numerical and experimental observations. Ravi-Chandar and Knauss [4] performed experiments of RCP in Homalite-100 and found that the velocity of a crack remains constant while the fracture surface shows a characteristic sequence of "mirror, mist and hackle" as the stress intensity factor increases. Based on the experimental observation they interpret that RCP in an amorphous, brittle solid is associated with multiple flaws or microcracks preceding the main crack and these multiple cracks coalesce to form the main crack.

In the present work we construct the two dimensional model of RCP inspired by the microcrack interpretation of RCP [4], and investigate the characteristic of the velocity of a crack for various different parameters. In section 2, the details of the model are described, and the fractal properties of the cluster of broken bonds at the plane of a crack is shown although they are not related with the fracture surface roughness. The fractal properties of this cluster are interesting by themselves, when the breaking rule is regarded as a rule which generate fractal patterns. Simulation results of the model of RCP are followed and discussion is given in section 3. 


\section{PERCOLATION MODEL OF RCP}

The plane of a crack, i.e., xy plane, is divided by the square lattice as shown in Fig. 1. The tensile stress is supposed to be applied in $z$ direction, which is perpendicular to xy plane. The bond between two atoms, aligned in $\mathrm{z}$ direction, is represented by each square of Fig. 1, and the distance between adjacent squares in xy plane is taken to be an unit distance. Suppose only one bond $A_{n}$ is broken at time $t=n$. Then we define that the bonds with the distance $\mathrm{R}\left(1 \leq \mathrm{R} \leq \mathrm{R}_{\mathrm{I}} \mathrm{C}\right)$ in $\mathrm{x}, \mathrm{y}$ direction could be broken at $t=n+1$ with the following probability $p$

$$
p=K / \sqrt{R} \text { for } R\left(1 \leq R \leq R_{I C}\right),
$$

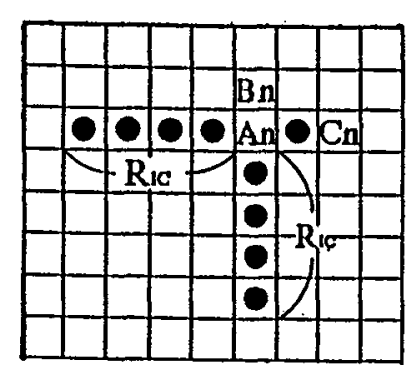

Fig. 1. Illustration of breaking rule of the model for RCP

where $K(0<K \leq 1)$ is a constant. The random number is generated with this breaking probability for each bond, which is located within the distance $R_{I C}$ from the bond $A_{n}$. When a bond is judged to be broken, the corresponding square is marked by the black color. Suppose bonds $A_{n}, B_{n}$ and $C_{n}$ are broken at time $t=n$ as shown in Fig. 1. The bonds with black circular mark represent the bond to be broken by the existence of the bond $A_{n}$. The bonds with the distance $R \leq R_{I C}$ in y direction beyond $B_{n}$ do not break because the bond $B_{n}$ is also broken and $A_{n}$ can not influence to them. These bonds, however, could be broken due to the existence of the bond $B_{n}$. The same rule is applied to the bonds beyond the bond $C_{n}$. The bond between $A_{n}$ and $C_{n}$ will be judged for breaking twice with the same probability $p=K$ by $A_{n}$ and $C_{n}$. The bonds $B_{n}$ and $C_{n}$ break the neighboring bonds with the same rule as $A_{n}$. The breaking rule of bonds is based on the following consideration. When a bond between atoms is broken, the stress wave is generated and propagates around it. The amplitude of the wave decreases as it propagates away, which is taken into account by the factor $1 / \sqrt{R}$ in Eq. (1). If the amplitude of the wave exceeds the critical breaking stress at the neighboring bond, this bond will be broken. The effect of the stress wave on breaking a bond varies due to thermal oscillation of the atom, which generates the probabilistic character of breaking. The propagation of a crack according to this breaking rule is thus regarded to be RCP.

\subsection{Fractal properties of the cluster of broken bonds}

The growth pattern of "fractured square lattice" starting from a single broken bond is shown in Fig. 2 . The black squares shown in Fig 2(a) show all of the broken bonds at certain instant. A cluster of broken bonds is defined as the connected region of the broken bonds in row and column of the square lattice. The broken bonds $A_{n}$ and $B_{n}$ shown in Fig. 1 form a cluster according to this rule. Fig. 2 (b) shows the cluster of broken bonds constructed from the broken bonds in Fig. 2 (a). The white region surrounded by the cluster of broken bonds is not connected with the cluster but some of the squares in this region, which could correspond to microcracks, are broken as shown in Fig. 2(a). The cluster of broken bonds corresponds to a crack. The external perimeter of the cluster is shown in Fig. 2 (c), in which the internal perimeters are eliminated. The external perimeter of the cluster of broken bonds for the various values of $K$ and $R_{I C}$ similar to Fig. 2 (c), are shown in Fig. 3. For the fixed value of $R_{I C \text {, }}$ $\mathrm{K}$ increases from 0.13 in Fig. 2(c) to 0.2 in Fig. 3(a), and then 0.6 in Fig. 3 (b). By comparing these three figures, we find that overhanging regions decrease as the value of $\mathrm{K}$ increases. The similar tendency can be noticed, though not so clear, by comparing Fig. 3(a) and (c). In this case the value of $\mathrm{K}$ 


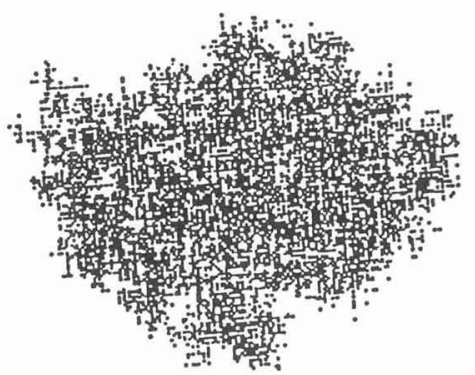

(a)

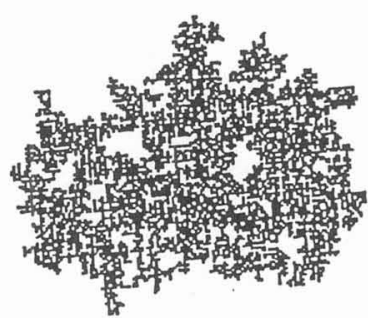

(b)

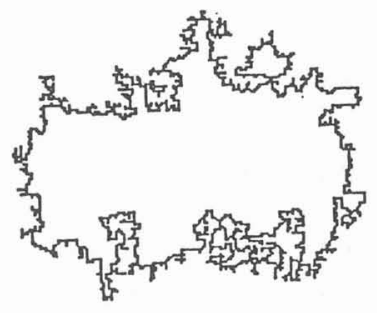

(c)

Fig. 2. The growth pattern of "fractured square lattice" starting from a single broken bond for the case $K=0.13, R_{1 C}=5$ is shown(a) All of the broken bonds are shown in black. (b) The cluster of broken bonds is shown in black. (c) The external perimeter of the cluster of the broken bonds is shown.

is fixed at $\mathrm{K}=0.2$, and the overhanging regions increase when $R_{I C}$ is increased markedly from Fig. 3 (a) to (c). Such tendency is captured by the value of fractal dimensions, $D_{f}$, which is measured by the method of box counting, i.e., the fractal dimension increases as overhanging regions increase. Starting from a single broken bond, four or five different cluster patterns of "fractured square lattice" are generated for fixed values of $K$ and $R_{I}$, and the fractal dimension of the external perimeter of these patterns are measured and shown in Fig. 4. As the value of $K$ becomes smaller for fixed value of $R_{I C}=5$, the fractal dimension increases on average as shown in Fig. 4(a). The fractal dimension of the cluster of broken bonds remains relatively constant for wide range of values of $R_{\mathrm{IC}}$ for the fixed value of

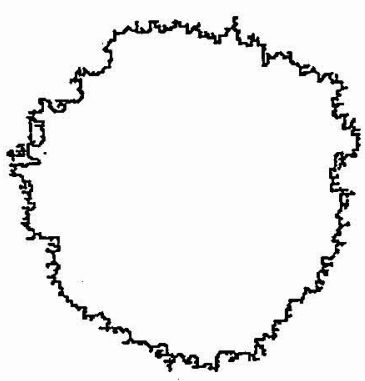

(a)

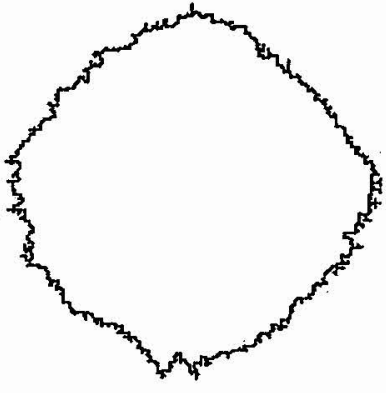

(b)

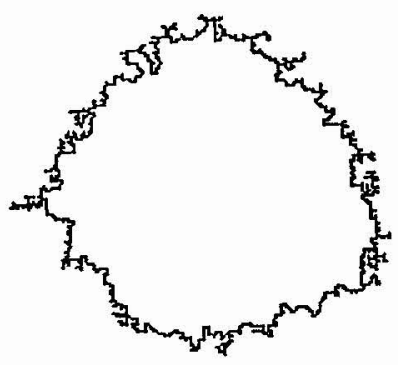

(c)

Fig. 3. The external perimeter of the cluster of "fractured square lattice" starting from a single bond.
(a) $\mathrm{K}=0.2, \mathrm{R}_{\mathrm{IC}}=5, \mathrm{D}_{\mathrm{f}}=1.16$
(b) $\mathrm{K}=0.6, \mathrm{R}_{\mathrm{IC}}=5, \mathrm{D}_{\mathrm{f}}=1.12$
(c) $\mathrm{K}=0.2, \mathrm{R}_{\mathrm{IC}}=20, \mathrm{D}_{\mathrm{f}}=1.18$

$\mathrm{K}=0.2$ as shown in Fig. 4 (b). These fractal properties of the cluster of "fractured square lattice" is not related with the fracture surface roughness since these pattern lies at the plane of a crack. The measured fractal dimension of the patterns range from 1.1 to 1.2. Theoretical analysis of these fractal dimensions is not possible at this moment since the present model is complex and quite different from the traditional percolation problem, which has been widely studied as a simple approach to critical phenomena in disordered system [5]. 


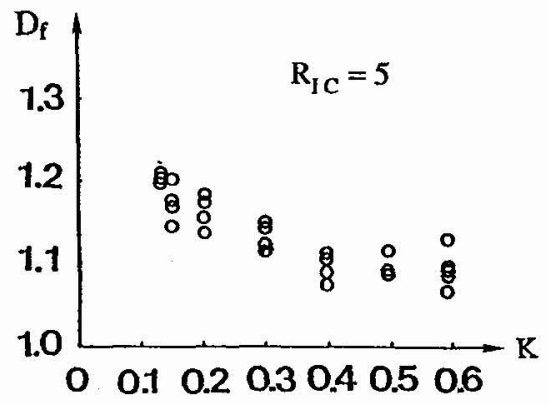

(a)

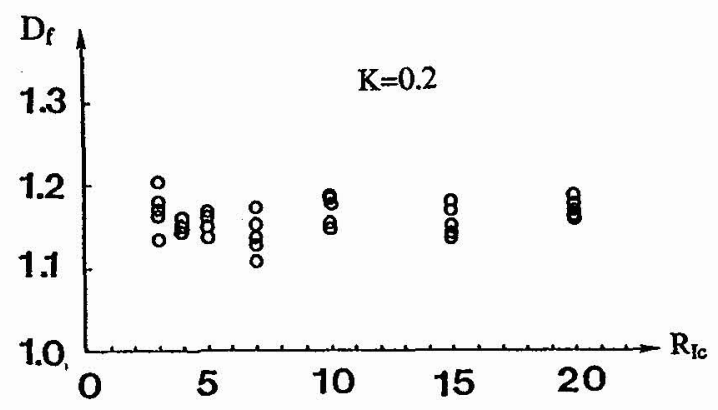

(b)

Fig. 4. Fractal dimensions of four or five different patterns of the external perimeter of "fractured square lattice", starting from a single broken bond, are shown. (a) The parameter, $R_{1 C}=5$, is fixed in this case. (b) $K=0.2$ is fixed.

\subsection{Simulation of RCP}

In order to simulate RCP, we define the plane of a crack OABC as shown in Fig. 5. The width of the plate, $\mathrm{OA}$ or $\mathrm{BC}$, is taken to be $\mathrm{L}_{\perp}=60$ and the length in the direction of RCP is $L_{o}=270$. Simulations of RCP shown from Fig. 6 to Fig. 10 are performed at these values of $L_{\perp}$ and $L_{0}$. The initial crack tip is defined as $O A$ and all bonds in the region ODEA could be broken at $\mathrm{t}=1$ according to the breaking rule. If the probability of breaking a bond is taken to be $p=1$ for $1 \leq R \leq R_{I}$, the crack propagates without changing the shape of the crack tip and the velocity, $C_{b}$, of a crack for this case is given by

$$
\mathrm{C}_{\mathrm{b}}=\mathrm{R}_{\mathrm{I} \mathrm{C}}
$$

When the probability of breaking a bond is given by Eq. (1), the shape of the crack tip changes as shown in Fig. 6 (c), which is defined as the front of the extemal perimeter of the cluster of broken bonds in the direction of crack propagation. Fig. 6 (a) and (b) correspond to Fig. 2 (a) and (b), respectively. In order to obtain the quantitative information of RCP, the number of broken bonds divided by $L_{\perp}$ at the position $x$ is defined as $p_{\text {open }}(x)$ and shown in Fig. 7 (b). The quantity $p_{f}(x)$ is equal to the number of broken bonds in the cluster divided by $L_{\perp}$ at $x$, where such fracture line of the crack tip as Fig. 6 (c) lies in this position $x$. The average position of the crack tip, $\mathbf{x}_{\text {tip, }}$ is defined as

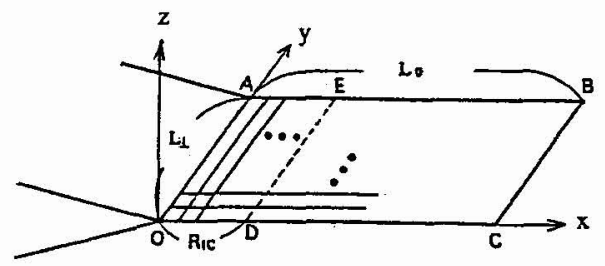

Fig. 5. The square lattice and the coordinate at the plane of a crack is shown. OA represents the initial crack tip.

(a)
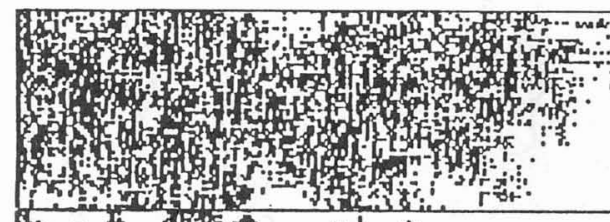

(b)
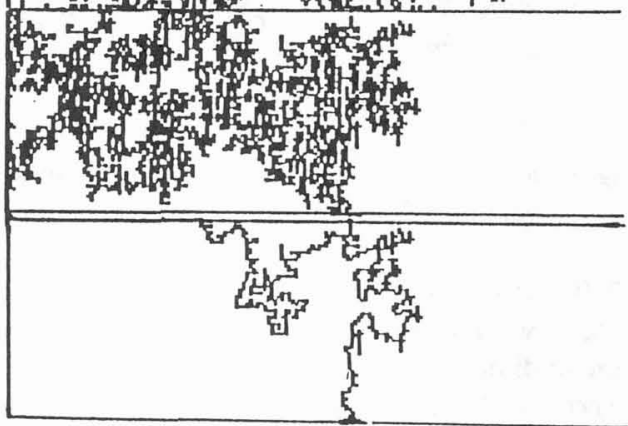

Fig. 6. The simulation for the case $R_{Y C}=6, K=1 / 4 \sqrt{5}$ (a) The black square represent all of the broken bonds. (b) The cluster of broken bonds is shown in black. (c) The front of the external perimeter of the cluster of broken bonds is shown. 


$$
x_{\text {tip }}=\int_{0}^{x_{0}} x p_{f}(x) d x
$$

and shown in Fig. 7 (b). The value of $x_{\text {tip }}$ at the instant, $t=n$, is defined as $x_{t i p}(n)$. The velocity of the crack tip at time $t(=n)$ is defined as

$$
\dot{a}(\mathrm{t})=\mathrm{x}_{\mathrm{tip}}(\mathrm{n})-\mathrm{x}_{\mathrm{tip}}(\mathrm{n}-1) .
$$

The velocity of the cluster of broken bonds, $\dot{A}$, is also defined in the following

$$
\dot{A}(t)=X_{\text {cluster }}(n)-X_{\text {cluster }}(n-1),
$$

where $X_{\text {cluster }}(n)$, shown in Fig. 7 (b), is equal to the number of broken bonds which belong to the cluster of broken bonds divided by $L_{\perp}$ at $t=n$. The velocities defined by Eqs, (4) and (5) versus $t$ are shown in Fig. 7 (c). These velocities could exceed $C_{b}$ by definition of these quantities. The velocity, $\dot{a}$, fluctuates as the shape of such crack front as Fig. 6 (c) changes. The fluctuation of the velocity, $\dot{a}$, is larger than that of $\dot{A}$ because the velocity, $\dot{A}$, is defined as the quantity averaged over the whole cluster of broken bonds, while $\dot{a}$ is defined as the average over the crack front. Figs. 8 and 9 show the results of the simulation of RCP by changing the parameters $K$ and $R_{I C}$, respectively. The value of $\dot{a} / C_{b}$ and $\dot{A} / C_{b}$ at each instant is shown as a dot in Fig. 8. All dots at fixed value of $\mathrm{K}$ in Fig. 8, for example, show that the normalized velocities $\dot{a} / \mathrm{C}_{\mathrm{b}}$ or $\dot{A} / C_{b}$ at each instant obtained in one such simulation as Fig. 7. The quantities, $\dot{a} / \mathrm{C}_{\mathrm{b}}$ and $\dot{A} / C_{b}$ at small values of $K$ seem to fluctuate so much because these velocities are rather slow and it takes more time to reach the end $\mathrm{BC}$ shown in Fig. 5. Besides, the fractal dimension of the cluster of broken bonds increases as is seen from Fig. 4 (a). The similar trends is observed for $\dot{a} / \mathrm{C}_{b}$ at small values of $R_{I C}$ in Fig. 9 (a), while the velocity $A_{\text {in Fig. }} 9$ (b) does not fluctuate. The difference

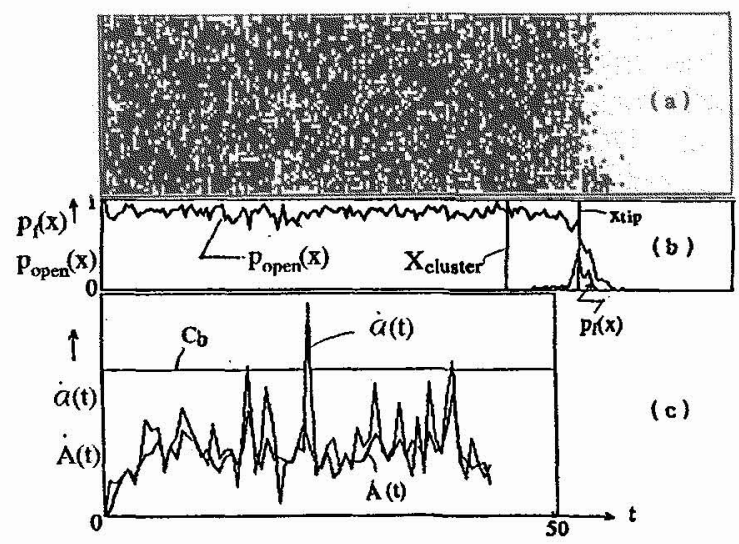

Fig. 7. The simulation of RCP for the case $R_{1 C}=5$ and $\mathrm{K}=0.25$. (a) All of the broken bonds are shown in black. (b) The quantities, $p_{\text {open }}(x), p_{f}(x), x_{\text {tip }}$ and $X_{\text {chuster, are }}$ shown. (c) The velocities, $\dot{a}(t)$ and $\dot{A}$, respectively defined in Eqs. (4) and (5), are shown as a function of time t.

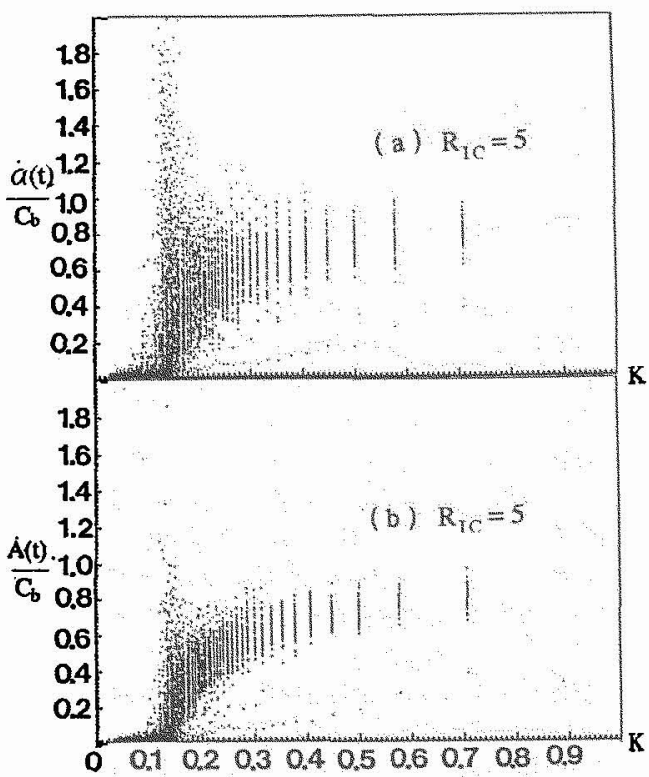

Fig. 8. The result of simulations for various different values of $K$ with fixed value of $R_{I C}=5$. (a) The quantity $\dot{a} / C_{b}$ at any instant of RCP for one simulation is shown as a dot at each value of $K$. (b) The quantity $\dot{A} / C_{b}$ at any instant of RCP for one simulation is also shown. 
between the two velocities $\dot{a}$ and $\dot{A}$ is reduced as the value of $K$ increases because the fractality of the cluster of broken bonds decreases. The simulation of RCP shown in Fig. 10 is performed in such parameter regime as $\mathrm{K}=0.5$ and $R_{\mathrm{IC}}=10$. Little difference between the velocities, $\dot{a}$ and $\dot{A}$, is observed in Fig. 10, and most bonds defined at each square lattice are broken. Therefore the crack front in this case is supposed to be smooth, which eliminate the difference between $\dot{a}$ and A. Notice that a few dots seen in low velocity region, shown in Fig. 8 and Fig. 9, comes from the initial velocity observed at time $t=1$. In order to see the effect of $\mathrm{L}_{\perp}$ on $\dot{a}$ and $\dot{\mathrm{A}}$, we have measured the average velocities $\langle\dot{a}\rangle$ and $<\dot{\mathrm{A}}>$ together with their fluctuations $<|\dot{a}-<\dot{a}>|>$ and $<|\dot{\mathrm{A}}-<\dot{\mathrm{A}}>|>$ for the case $R_{I C}=5$ and $K=0.25$, as shown in Fig. 11 . The bracket $<>$ represents time average of the quantity over one simulation. The average velocities $\langle\dot{a}\rangle$ and $\langle\dot{A}\rangle$ are found to be $<\dot{a}\rangle \approx\langle\dot{A}\rangle \approx 0.55 \mathrm{C}_{b}$ independent of $\mathrm{L}_{\perp}$, while the fluctuations, \langle|$\dot{a}-\langle\dot{a}>|>$ and $<|\dot{A}-<\dot{A}>|>$ decrease as $L_{\perp}$ increases.

\section{DISCUSSION}

Quantitative comparison between the present simulation and the experiment of RCP is not possible since the sound wave, which plays an important role in RCP, does not exist in the simulation. The velocity, $\mathrm{C}_{\mathrm{b}}$, is nothing to do with the velocity of sound wave. The probabilistic aspects of the model, however, exist in such mechanisms of RCP as generation and coalescence of microcracks at the tip of a crack [4]. The generation and coalescence of microcracks is closely correlated with various defects of the materials, which distribute at random. Therefore the present model takes partial account of the real experiment. In order to compare the results of the present simulation with the real experiment, we consider the temporal and spatial correspondence between them. The time scale of breaking a bond between atoms is much smaller than the temporal precision of the measurement of the velocity of a crack. The macroscopic measurement of the crack velocity in experiments corresponds to the time averaged velocity of breaking bonds in simulation. In other words, the velocities $\dot{a}$ or $\dot{\mathrm{A}}$ observed in the simulations shown from 
Fig. 7 to Fig. 10 do not correspond to the velocity of RCP and only the time averaged velocities $\langle\dot{a}\rangle$ and $\langle\dot{A}\rangle$ in Fig. 11, which we find a constant, are of practical interest. As to the spatial scale of the simulation, the size of the square lattice correspond to the atomic scale of the material. Since the width of the plate in the experiment is of macroscopic scale, the results of simulation are meaningful in the limit of $\mathrm{L}_{\perp} \rightarrow \infty$, of which information can be obtained from Fig. 11. We find from Fig. 11 that the fluctuation of the normalized velocities, $\dot{a} / C_{b}$ and $\dot{A} / C_{b}$, is negligible and these velocities take the constant value, $\langle\dot{a}\rangle \approx\langle\dot{\mathrm{A}}\rangle \approx 0.55 \mathrm{C}_{\mathrm{b}}$. Since the amplitude of the sound wave is essentially of macroscopic concept, it is more appropriate to choose relatively large values for $R_{I C}$, in which regime the velocities, $\dot{a} / \mathrm{C}_{\mathrm{b}}$ and $\dot{\mathrm{A}} / \mathrm{C}_{\mathrm{b}}$, also tend to be a constant. We thus conclude that the present simulation of RCP predicts qualitatively that the velocity of a crack become constant, in agreement with the experiment [3], when the fracture surface is smooth under the restrictions discussed above.

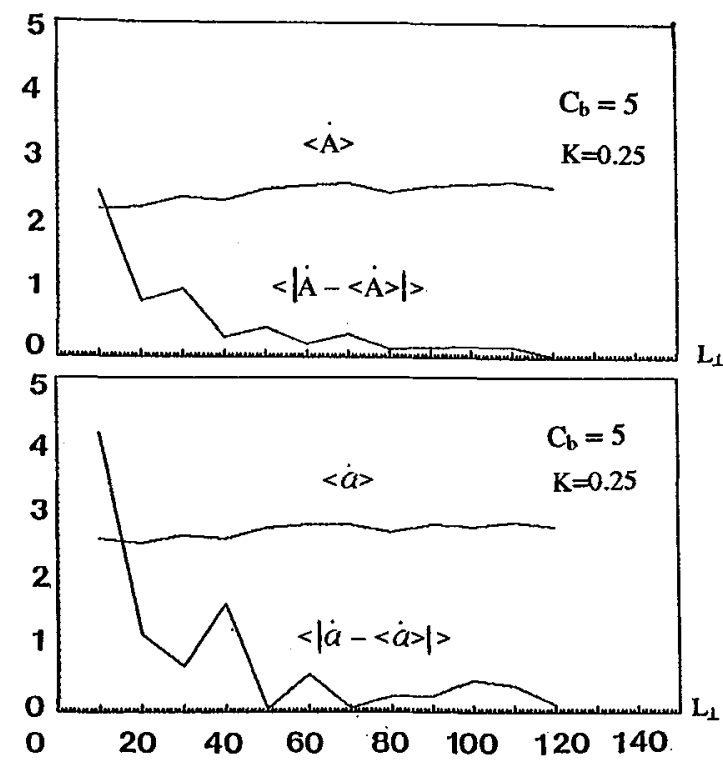

Fig. 11 Time average and it's fluctuation of the velocities $\dot{A}$ and $\dot{\alpha}$ are shown as a function of $L_{\perp}$ for the case $R_{\text {IC }}=5$ , $K=0.25$ and $L_{o}=270$. (a) $<\dot{A}>$ and $<|\dot{A}-<\dot{A}>|>$ versus $\mathrm{L}_{\perp}$ are shown. (b) $\left\langle\dot{a}>\right.$ and \langle|$\dot{a}-\langle\dot{a}>|>$ versus $L_{\perp}$.

\section{References}

[1] Fineberg J., Gross S.P., Marder M. and Swinney H.L., Phys. Rev. Lett. 67 (1992) 457-460 Phys. Rev. B45 (1992) 5146-5154

[2] Sharon E., Gross S.P. and Fineberg J., Phys. Rev. Lett. 74 (1995) 5096-5099

[3] Ivankovic A. and Williams J.G., Int. J. of Fracture, 64 (1993) 251-268

[4] Ravi-Chandar K. and Knauss W.G., Int. J. of Fracture 26 (1984)141-154

[5] Stauffer D., Phys. Reports 54 (1979) 1 